\title{
Concentración de selenio en leche de estanques de rebaños lecheros del sur de Chile y su relación con indicadores productivos
}

\author{
Bulk tank milk selenium concentration in dairy herds from Southern Chile and its \\ relationship to productive performance

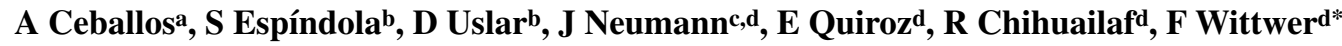 \\ aGrupo de Investigación en Biología de la Producción, Universidad de Caldas, Manizales, Colombia. \\ bCooperativa Agrícola y Lechera de La Unión, La Unión, Chile. \\ cUniversidad Católica de Temuco, Chile, \\ dnstituto de Ciencias Clínicas Veterinarias, Facultad de Medicina Veterinaria, \\ Universidad Austral de Chile, Valdivia, Chile.
}

\begin{abstract}
SUMMARY
An observational study was conducted in 59 Chilean dairy farms to evaluate bulk tank milk selenium (Se) concentration, and its association with measures of milk production parameters and udder health, measured as bulk tank somatic cell count. Fifty-nine dairy herds from Southern Chile were selected. Bulk tank milk samples were collected four times (Spring 2007 and Summer, Autumn and Winter 2008). A survey about general and nutritional management of the herds was conducted. Data on productive performance were also collected. Data were analyzed by using linear mixed models. Mean bulk tank milk Se concentration was $14.7 \mu \mathrm{g} / \mathrm{L}$ (SD: $6.5 \mu \mathrm{g} / \mathrm{L}$ ), indicating a high variation among herds (44\%). No association between bulk tank milk Se concentration and herd productive performance was observed. Nevertheless, $82 \%(\mathrm{n}=231)$ of bulk tank milk samples had a milk Se concentration lower than the reference of $20 \mu \mathrm{g} / \mathrm{L}$. These results are indicating that bulk tank milk Se concentration in selected dairy herds from Southern Chile is lower than international reference values, reflecting an inadequate intake of Se. This inadequacy was not necessarily translated into low milk yield or low quality milk.
\end{abstract}

Palabras clave: bovinos lecheros, leche, estanque de frío, selenio.

Key words: dairy cattle, milk, bulk tank, selenium.

\section{INTRODUCCIÓN}

La leche contribuye a satisfacer los requerimientos nutricionales básicos de la población humana (Bauman y col 2006). Así, una porción de leche (100g/día) puede aportar al menos el 10\% del requerimiento diario de un nutriente en particular; considerándose una buena fuente si su aporte es cercano al $25 \%$. Además del aporte de nutrientes, la leche es fuente de moléculas bioactivas (moléculas con actividad biológica) que han sido asociadas con beneficios para la salud humana (Bauman y col 2006), señalándose desde propiedades anticarcinogénicas hasta la prevención de enfermedades cardiovasculares cuando se consume regularmente (Pfeuffer y Schrezenmeir 2000).

La justificación para modificar la composición nutricional de la leche obedece a tres razones (Knowles y col 2004): 1) Mejoramiento del proceso industrial para la producción de derivados lácteos, 2) Alterar su valor nutricional para ajustarse a guías dietéticas determinadas por agencias gubernamentales, y 3) Usar la leche como

Aceptado: 15.11.2012.

* Casilla 567, Valdivia, Chile; fwittwer@uach.cl un sistema de entrega de nutracéuticos con un conocido beneficio para la salud humana.

El selenio (Se), micronutriente esencial en la alimentación humana, está presente en la leche. La leche derivada de vacas en pastoreo ofrece una fuente de Se que es altamente biodisponible para el ser humano debido a la fuente del mineral, contribuyendo sustancialmente a satisfacer el requerimiento diario de Se para una persona (Knowles y col 2004). El consumo de $100 \mathrm{~g}$ de leche por día puede proveer al menos un $10 \%$ del Se requerido diariamente por personas adultas siempre que la concentración de Se en la leche sea mayor a $30 \mu \mathrm{g} / \mathrm{L}$ (Knowles y col 2004). Además, se ha recomendado que las fórmulas infantiles basadas en leche provean como mínimo $10 \mu \mathrm{g}$ de Se/día para complementar la leche materna (Carver 2003). Consecuentemente, los derivados lácteos enriquecidos con nutrientes específicos son un concepto novedoso y atractivo para la promoción de una mejor salud humana (Knowles y col 1999, Knowles y col 2004).

En el sur de Chile se ha descrito la carencia de Se en forrajes (Ceballos y col 1999, Wittwer y col 2002), lo que ha llevado a que los rumiantes en pastoreo en esta zona tengan una baja actividad sanguínea de la enzima antioxidante glutatión peroxidasa (GPx1; EC 1.11.1.9) 
(Ceballos y col 1998). Por otro lado, los requerimientos para bovinos en crecimiento y lactancia son mayores (NRC 2001), lo que lleva a la necesidad de otorgar una mayor atención en el manejo nutricional de los animales a riesgo, o que por razones de manejo su suplementación mineral sea insuficiente, como las vaquillas.

En el sur de Chile, hasta un $25 \%$ de las vaquillas y un $17 \%$ de las vacas en lactancia tuvieron una actividad de GPx1 compatible con un bajo aporte dietario de Se (Ceballos y col 1998, Laporte 1998, Wittwer y col 2002). Esta carencia ha sido asociada con diversas alteraciones patológicas en bovinos, tales como retención de placenta, infertilidad, baja producción de leche, reducción en la tasa de crecimiento, alteraciones de la respuesta inmune y reducción de la resistencia a enfermedades (Wichtel 1998). Respecto al mecanismo de acción, el Se junto a la vitamina $\mathrm{E}$, parecen actuar en forma concertada como antioxidantes celulares, pudiendo tener cada uno un efecto moderado sobre el otro elemento en cuanto a la protección sobre algunas enfermedades, como retención de placenta y mastitis (Wichtel 1998).

Los suplementos con Se han sido utilizados para mejorar su balance en vacas lecheras y con ello incrementar el contenido en la leche (Knowles y col 1999, Grace y col 2001, Ceballos y col 2009). Estudios realizados algunos años atrás indicaron que una baja proporción del Se era transferida a la leche después de usar formas inorgánicas como selenito de sodio, aumentando cuando la fuente provenía de fuentes naturales (e.g. forrajes) (Conrad y Moxon 1979). Estos autores concluyeron que el Se proveniente de fuentes naturales podía ser transferido más fácilmente a la leche debido a una mayor disponibilidad. Además, se observó que un incremento en el consumo de Se no produciría un efecto marcado en la leche de vacas alimentadas con una ración base adecuada en su contenido de $\mathrm{Se}$, que correspondería a $>0,1 \mathrm{mg} / \mathrm{kg}$ base materia seca.

Se ha descrito previamente la relación entre el Se sanguíneo y en la leche (Knowles y col 1999). Estos resultados apoyan el uso de la leche como una herramienta diagnóstica para monitorear el estado de Se en vacas individualmente y además en muestras de leche obtenidas de estanque de frío que pueden usarse para el seguimiento a nivel de grupo o de rebaño (Grace y col 2001, Wichtel y col 2004, Ceballos y col 2009). El análisis de Se en leche de estanque de frío puede usarse como una prueba tamiz para el análisis del balance de Se en el rebaño, donde muestras con valores inferiores a $35 \mu \mathrm{g} / \mathrm{L}$ requerirían un detallado análisis al nivel de la vaca (Grace y col 2001). En un estudio realizado en la Isla Príncipe Eduardo, Canadá, el $59 \%$ de los rebaños fueron clasificados según su contenido de Se en leche de estanque de frío como marginalmente carentes en Se o con una carencia real, presentándose variaciones significativas según la época del año y cuyos valores estaban relacionados con la cantidad y calidad del Se suplementado (Wichtel y col 2004).
La respuesta a las diferentes fuentes de Se y la transferencia del Se a la leche requieren la realización de estudios con el objetivo de determinar su eficacia en términos del contenido de Se en la leche y el mantenimiento de una concentración estable en el tiempo. Adicionalmente, después de la realización de diferentes estudios observacionales con respecto al balance de Se en rebaños lecheros en el sur de Chile, se han introducido modificaciones en el manejo de la suplementación mineral sin que se haya estudiado el impacto de esta suplementación sobre indicadores productivos en el rebaño. Así, se ha planteado como hipótesis para este estudio que el contenido de Se en la leche obtenida de rebaños mantenidos en condiciones pastoriles del sur de Chile está acorde con los requerimientos internacionales y que sus variaciones se asociarían a factores propios de cada predio y manejo del rebaño. Con esta información se pueden diseñar estrategias futuras de suplementación para rebaños en pastoreo o semiconfinamiento, para producir productos lácteos de alta calidad para consumo humano, y para responder a un mercado creciente de productos enriquecidos demandados por aspectos de salud y estilo de vida. El objetivo de este estudio fue establecer la concentración de Se en la leche de estanques de frío en rebaños lecheros del sur de Chile y su relación con indicadores de producción y el recuento de células somáticas.

\section{MATERIAL Y MÉTODOS}

\section{REBAÑOS}

Fueron seleccionados 59 rebaños de productores lecheros que entregaban su producción a la planta industrializadora de leche de la Cooperativa Agrícola y Lechera de la Unión, COLUN, ubicada en la Región de Los Ríos, Chile. La selección se realizó mediante un muestreo dirigido según el sistema productivo y el sistema de alimentación, igualmente se consideró el volumen anual de leche comercializado y la disposición para permitir la obtención de las muestras. Los rebaños estaban localizados en distintas zonas geográficas, identificadas por macrozonas 4, 5, 7 y 8 según la definición del Consorcio Lechero de Chile (cuadro 1). Estos rebaños entregaron aproximadamente un $41 \%$ del volumen total de leche recibida en planta en la primavera de 2007.

\section{MUESTRAS}

De cada rebaño seleccionado para el estudio se obtuvo una muestra de leche del estanque de frío en la primavera (octubre-noviembre) de 2007, en el verano (febrero), otoño (mayo) y final del invierno (septiembre) de 2008. Las muestras se congelaron $\left(-20^{\circ} \mathrm{C}\right)$ hasta la posterior determinación de la concentración de Se en el Laboratorio de Patología Clínica Veterinaria de la Universidad Austral de Chile.

Igualmente, se recolectó información general de cada uno de los predios mediante una encuesta que contenía 
Cuadro 1. Distribución por macrozona lechera de 59 predios lecheros encuestados para determinar la concentración de selenio en leche de estanques en el sur de Chile ${ }^{1}$.

Distribution of dairy herds according to milk production macro zones used to determine the selenium content in bulk milk in the south of Chile.

\begin{tabular}{llr}
\hline Macrozona & Sector & $\begin{array}{c}\mathrm{N}^{\mathbf{0}} \text { de } \\
\text { predios }\end{array}$ \\
\hline 4, Llano Central, Región XIV, de Los Ríos & Lanco, Máfil, Los Lagos, Paillaco & 21 \\
5, Llano Central, Región X, de Los Lagos & Río Bueno, Osorno, Purranque, Casma & 19 \\
7, Precordillera Andina, Regiones IX, XIV y X & Villarrica, Panguipulli, Futrono, Lago Ranco, Puyehue, Rupanco, Pto. Octay & 10 \\
8, Precordillera Costa, Regiones XIV y X & La Unión, Costa Osorno, Río Negro, Crucero, Fresia & 9 \\
\hline
\end{tabular}

1 www.consorciolechero.cl/chile/pags/macro-zonas.php

información con respecto al manejo general del hato, sistema de producción, número de vacas en lactancia, producción de leche, manejo nutricional, uso o no de suplementos minerales, suplementación o no con Se, método de suplementación y cantidad suplementada, si correspondía. Las encuestas fueron aplicadas directamente en el predio mediante entrevista con el propietario del mismo, o con el veterinario asesor en caso de no ser posible la entrevista con el propietario. La información con respecto al contenido de grasa, proteína (Foss Milkoscan) y recuento de células somáticas en el estanque (Fossomatic) se obtuvo directamente de la planta lechera. Igualmente, se recolectaron aproximadamente $300 \mathrm{~g}$ del forraje probablemente pastoreado (i.e. no se realizó corte a ras del suelo) por las vacas durante la primavera de 2007 para determinar la concentración de Se.

\section{ANÁLISIS}

La concentración de Se en las muestras de leche se determinó mediante espectrofotometría de absorción atómica con generación de hidruros. Las muestras, previa descongelación a baño temperado $\left(37^{\circ} \mathrm{C}\right)$, fueron digeridas según un protocolo de dos etapas y luego cuantificadas (Muñiz-Naveiro y col 2005). Para la digestión, en una primera etapa se pesaron $2,5 \mathrm{~g}$ de leche entera homogenizada, se le adicionaron $2 \mathrm{~mL}$ de ácido nítrico $1 \mathrm{~mL}$ de peróxido de hidrógeno $(30 \%)$ y $2,5 \mathrm{~mL}$ de agua deionizada. Las muestras fueron llevadas a un horno microondas Milestone modelo Start D con rotor de 12 posiciones MRD/12S, y sometidas a un programa de calentamiento por $10 \mathrm{~min}$ a $200{ }^{\circ} \mathrm{C}$ y de mantenimiento por $10 \mathrm{~min}$ a $200^{\circ} \mathrm{C}$, luego se dejaron enfriar $\mathrm{a}<50^{\circ} \mathrm{C}$, donde se agregaron $1,4 \mathrm{~mL}$ de ácido clorhídrico. Posteriormente, las muestras fueron sometidas a un programa por $5 \mathrm{~min}$ a $130^{\circ} \mathrm{C}$ y de mantenimiento por $10 \mathrm{~min}$ a $130^{\circ} \mathrm{C}$. Luego las muestras fueron enfriadas a $<50^{\circ} \mathrm{C}$ y se les agregó $0,4 \mathrm{~mL}$ de urea $(50 \%$ $\mathrm{m} / \mathrm{v}$ ) y se aforaron a $20 \mathrm{~mL}$.

La determinación de la concentración de Se fue realizada con un espectrofotómetro de absorción atómica Thermo Electron Solar S, a 196 nm, con aditamento de generación de hidruros (Thermo Scientific VP100), expresando los resultados en $\mu \mathrm{g} / \mathrm{L}$. Para la calibración del equipo se contó con un patrón de Se con una concentración de 1000 ppm (Merck), del que se obtuvo una dilución de 10 ppm con la que se elaboró una nueva dilución de 500 ppb, para así a partir de ella lograr los tres estándares finales de 5,10 y $20 \mathrm{ppb}$, que junto con una muestra blanco se utilizaron para calibrar el equipo. Después de la lectura de 12 muestras consecutivas se procedió a recalibrar el equipo con un estándar y una muestra blanco.

La determinación de la concentración de Se en las muestras de forrajes se realizó con un procedimiento similar basado en un secado previo de las muestras y digestión húmeda con ácido nítrico y peróxido de hidrógeno, seguido de la determinación de la concentración de Se con un espectrofotómetro de absorción atómica (Thermo Electron Solar S) a una longitud de onda de $196 \mathrm{~nm}$ con aditamento de generación de hidruros (Thermo Scientific VP100). Las determinaciones se realizaron en duplicado expresando los resultados en $\mu \mathrm{g} / \mathrm{L}$.

Una concentración $<9,5 \mu \mathrm{g} / \mathrm{L}$ fue considerada como carencial, entre 9,5 y 20,0 $\mu \mathrm{g} / \mathrm{L}$ como marginal, y > 20,0 $\mu \mathrm{g} / \mathrm{L}$ como adecuada. Esta referencia se estableció mediante una regresión lineal entre la media para la concentración sérica de Se con la concentración de Se en la leche del estanque en 15 rebaños lecheros en la Isla Príncipe Eduardo en Canadá (Wichtel y col 2004). Los puntos de corte para la concentración de Se en la leche del estanque fueron seleccionados mediante la obtención del punto donde la concentración sérica de referencia intercepta la línea de regresión del modelo (Wichtel y col 2004).

\section{ANÁLISIS ESTADÍSTICO}

Este estudio correspondió a uno de tipo longitudinal caracterizado por tener varias mediciones consecutivas (e.g. una muestra por cada estación del año) en el mismo estanque de enfriamiento. Un total de 231 muestras de leche fueron colectadas para la evaluación de la concentración de Se en leche. Se utilizó un modelo de regresión lineal mixto considerando el predio como 
variable aleatoria y agrupada dentro de la zona geográfica (macrozona lechera) para evaluar la concentración de Se en el estanque de frío (Dohoo y col 2009). Las variables independientes evaluadas fueron: región geográfica (e.g. macrozonas lecheras 4, 5, 7 y 8), época del año (e.g. primavera 2007, verano, otoño e invierno 2008), tamaño del rebaño, concentración de Se en la pradera, sistema de producción (e.g. pastoreo, confinamiento, mixto) y uso o no de suplementación mineral con Se adicional a la mezcla de minerales en la ración. Posteriormente, la variable concentración de Se en leche se utilizó como variable independiente para establecer la eventual relación con los indicadores de producción, para lo cual se utilizaron también modelos de regresión lineal mixtos (Dohoo y col 2009).

Los supuestos del modelo fueron evaluados mediante el análisis de los residuos estandarizados. Para aproximar el recuento de células somáticas (RCS) en el estanque a la distribución normal, se utilizó una transformación logarítmica de los valores de RCS en el estanque (1000 células/ mL) (Ali y Shook 1980). Los análisis fueron realizados usando el procedimiento MIXED en SAS versión 9.2 (SAS Institute Inc., Cary, NC, USA).

\section{RESULTADOS}

El sistema de producción en la mayoría de los rebaños estaba basado en el pastoreo (80\%). De ellos, un $41 \%$ indicaron confinar de alguna forma las vacas durante el invierno. Un menor número de predios tenía pastoreo estacional $(7 \%)$ o confinamiento permanente $(3,4 \%)$ como sistemas de producción. En el cuadro 2 se presentan los resultados para las variables productivas en la primavera de 2007.

Con respecto a la suplementación mineral, un $88 \%$ de los predios encuestados utilizaba suplementos minerales durante todo el año, siendo su incorporación en el concentrado el método preferido (32\% de los predios), seguido por el suministro directo en el comedero (19\%). Un $41 \%$ de los rebaños estudiados no indicaron el método de suplementación mineral, siendo un porcentaje adecuado para el sesgo de no respuesta en estudios observacionales (Dohoo y col 2009).

La suplementación con Se era una práctica rutinaria en el $61 \%$ de los rebaños. De ellos un $27 \%$ lo hacía especialmente para las vacas en el preparto mediante aplicación parenteral y en el 5\% de los rebaños se suministraba Se suplementario en forma oral. No fue posible establecer si la suplementación con Se era una práctica rutinaria en un $29 \%$ de los rebaños encuestados.

\section{CONCENTRACIÓN DE SELENIO EN FORRAJES}

En total se analizaron 55 muestras de forrajes provenientes de los diferentes predios, las que correspondían a praderas de Ryegrass (Lolium spp), perennes y de rotación, con o sin trébol blanco o rosado (Trifolium spp) en la mayoría de los casos. La concentración media de Se en el forraje fue $0,07 \mathrm{mg} / \mathrm{kg}$ base seca, observándose una variación cercana al $90 \%$ en la concentración del mineral. Se observaron variaciones entre $0,01 \mathrm{y}$ $0,28 \mathrm{mg} / \mathrm{kg}$ base seca. En total, un $43 \%$ de las muestras analizadas presentaron valores inferiores a $0,05 \mathrm{mg} / \mathrm{kg}$, si bien no se presentaron diferencias entre las zonas geográficas estudiadas $(\mathrm{P}=0,85)$.

\section{CONCENTRACIÓN DE SELENIO EN LECHE}

La concentración media de Se en la leche del estanque fue $14,7 \mu \mathrm{g} / \mathrm{L}$ (DE: $6,50 \mu \mathrm{g} / \mathrm{L}$ ), con una variación entre 2,5 y $46,3 \mu \mathrm{g} / \mathrm{L}$. En total se observaron 47 valores inferiores a $9,5 \mu \mathrm{g} / \mathrm{L}(20 \%)$, mientras que 143 observaciones (60\%) presentaron valores entre 9,5 y $20,0 \mu \mathrm{g} / \mathrm{L}$. Los valores bajos y marginales fueron observados en todas las zonas geográficas del estudio sin el predominio de una en particular (cuadro 3).

Cuadro 2. Índices de producción en 59 rebaños lecheros del Sur de Chile durante la primavera de 2007. Production index obtained from 59 dairy herds of the south of Chile during spring 2007.

\begin{tabular}{lccccc}
\hline Variable & $\mathrm{n}^{1}$ & Media & DE & Mediana & Intervalo \\
\hline Vacas en lactancia & 53 & 411 & 331 & 300 & $59-1802$ \\
Días en leche & 30 & 178 & 26 & 178 & $130-229$ \\
Media de producción (kg/vaca/d) & 46 & 26,2 & 3,8 & 26,3 & $18,9-32,9$ \\
Grasa en leche $(\%)^{2}$ & 58 & 3,52 & 0,36 & 3,49 & $2,56-4,96$ \\
Proteína en leche $(\%)^{2}$ & 58 & 3,43 & 0,14 & 3,43 & $3,04-3,91$ \\
RCS en tanque (1000 células/mL) 2,3 & 56 & 209 & 78 & 195 & $103-654$ \\
Intervalo parto-primer servicio (días) & 26 & 84 & 18 & 84 & $45-141$ \\
Intervalo parto-concepción (días) & 26 & 124 & 23 & 128 & $81-163$ \\
\hline
\end{tabular}

Número de predios que respondieron a la encuesta.

Información obtenida de la planta de recepción de leche (COLUN).

RCS: Recuento de células somáticas. 
Cuadro 3. Número de muestras de leche con valores carenciales $(<9,5 \mu \mathrm{g} / \mathrm{L})$ y marginales $(9,5$ a $20,0 \mu \mathrm{g} / \mathrm{L})$ para la concentración de selenio de un total de 231 muestras de tanque obtenidas de 59 predios lecheros ubicados en las macrozonas lecheras $4,5,7$ y 8 del sur de Chile.

Number of milk samples indicating a selenium deficiency $(<9.5 \mu \mathrm{g} / \mathrm{L})$ or marginal $(9.5$ to $20,0 \mu \mathrm{g} / \mathrm{L})$ balance from 231 bulk samples obtained from 59 dairy herds located within the milk production macro zones $4,5,7$ and 8 of the south of Chile.

\begin{tabular}{llrr}
\hline & Sector & \multicolumn{2}{c}{$\mathrm{N}^{\circ}$ de predios } \\
\cline { 2 - 4 } Macrozona & & Carencia & Marginal \\
\hline 4, Llano Central, Región XIV, de Los Ríos & Lanco, Máfil, Los Lagos, Paillaco & 18 & 50 \\
5, Llano Central, Región X, de Los Lagos & Río Bueno, Osorno, Purranque, Casma & 13 & 46 \\
7, Precordillera Andina, Regiones XIV y X & Villarrica, Panguipulli, Futrono, Lago Ranco, Puyehue, Rupanco, Octay & 9 & 25 \\
8, Precordillera Costa, Regiones XIV y X) & La Unión, Costa Osorno, Río Negro, Crucero, Fresia & 7 & 22 \\
\hline
\end{tabular}

No se observaron diferencias con respecto a las variables: zona geográfica $(\mathrm{P}=0,86)$; sistema de producción (pastoreo vs. confinamiento total o parcial, $\mathrm{P}=0,65)$; tamaño del rebaño (número de vacas en lactancia, $\mathrm{P}=0,98$ ); concentración de Se en el forraje $(\mathrm{P}=0,53)$, y suplementación con Se $(\mathrm{P}=0,26)$. Pese a lo anterior, hubo diferencias según la época del año, presentándose la concentración más baja en el verano 2008 (cuadro 4).

Las mayores concentraciones de Se en la leche del estanque se observaron en la primavera de $2007(17,7 \mu \mathrm{g} / \mathrm{L})$ y el otoño e invierno de 2008 ( $\mathrm{Se}=16,5$ y $16,1 \mu \mathrm{g} / \mathrm{L}$ respectivamente) (cuadro 4), las que fueron superiores a la concentración observada en el verano 2008, que presentó el valor más bajo obtenido en el estudio $(\mathrm{Se}=11,7 \mu \mathrm{g} / \mathrm{L})$ $(\mathrm{P}<0,01)$ coincidente, según la información entregada, con una menor suplementación con fuentes orgánicas de Se en algunos de los predios.

\section{CONCENTRACIÓN DE SELENIO EN LECHE E INDICADORES PRODUCTIVOS}

La evaluación incondicional de la concentración de Se en la leche con los indicadores productivos evaluados no fue significativa, excepto por la concentración de proteína láctea (cuadro 5).

Cuadro 4. Modelo de regresión para la asociación entre la concentración de selenio $(\mu \mathrm{g} / \mathrm{L})$ en la leche del estanque y la época del año en 59 lecherías del sur de Chile.

Regression model for the association between bulk milk selenium concentration $(\mu \mathrm{g} / \mathrm{L})$ and season of the year in 59 dairy herds from southern Chile.

\begin{tabular}{lclc}
\hline Efecto & Coef. $^{1}$ & IC al 95\% & Valor de P \\
\hline Intercepto & 16,11 & 14,$48 ; 17,74$ & $<0,01$ \\
Época del año & & & $<0,01$ \\
$\quad$ Primavera 2007 & $-1,41$ & $-3,48 ; 0,66$ & \\
$\quad$ Verano 2008 & $-4,44$ & $-6,49 ;-2,38$ & \\
$\quad$ Otoño 2008 & 0,34 & $-1,75 ; 2,43$ & \\
$\quad$ Invierno 2008 & 0 & & \\
\hline & & & \\
2 & Coeficiente. & &
\end{tabular}

Cuadro 5. Asociación incondicional de la concentración de selenio en la leche del estanque con diferentes indicadores de producción en 59 lecherías del sur de Chile durante la primavera 2007 y el verano 2008 .

Unconditional association of the bulk milk selenium concentration with production parameters of 59 dairy herds of the south of Chile, spring 2007 and summer 2008.

\begin{tabular}{lc}
\hline Respuesta & Valor de P \\
\hline Volumen de leche entregada en planta & 0,57 \\
Grasa láctea en \% & 0,85 \\
Proteína láctea en \% & 0,02 \\
RCS en estanque & 0,40 \\
\hline
\end{tabular}

La asociación entre la concentración de Se en el estanque y los parámetros productivos fue controlada por la época del año. La concentración de Se en la leche del estanque no afectó los indicadores productivos, observándose además que el efecto sobre proteína láctea no fue significativo $(\mathrm{P}=0,70)$, lo que indica que la relación entre el balance de Se, evaluado mediante la concentración de Se en la leche del estanque, y la proteína láctea estaba confundida por la época del año.

\section{DISCUSIÓN}

La concentración de Se en la leche del estanque responde rápidamente a los cambios en el consumo de $\mathrm{Se}$, siendo una herramienta útil para establecer el estado nutricional de Se en la lactancia (Wichtel y col 2004). Considerando como referente una concentración en leche $>20 \mu \mathrm{g} / \mathrm{L}$, el $80 \%$ de las muestras a lo largo de las zonas geográficas estudiadas presentó valores entre deficitarios o marginales. Lo que estaría explicado parcialmente por la baja concentración de Se en las praderas $(<0,05 \mathrm{mg} / \mathrm{kg}$ base materia seca en un $43 \%$ de las muestras), ya que el pastoreo era el sistema preferido en un $80 \%$ de los rebaños.

La leche del estanque de rebaños lecheros en una provincia canadiense, caracterizada por valores bajos de Se en las praderas, tenía valores entre 18,0 y $26,8 \mu \mathrm{g} / \mathrm{L}$ (Wichtel y col 2004). Esta concentración puede variar debido a diversos factores (Norrman 1984, Miller y 
col 1995, Wichtel y col 2004). Aunque se ha reportado una menor concentración en los meses de otoño e invierno (Wichtel y col 2004), los resultados de este estudio indicaron valores más bajos en la época de verano, coincidente con lo observado en Estados Unidos (Miller y col 1995) y Suecia (Norrman 1984). Variaciones estacionales en la concentración de Se en las praderas y diferentes cantidades de concentrado suministrado a las vacas según la época del año podrían explicar parcialmente los cambios estacionales en la concentración de Se en la leche del estanque. Asimismo, parte de la variación podría estar explicada por el sistema productivo objeto de los rebaños estudiados, ya que los partos están concentrados en dos épocas del año, primavera y otoño, consecuentemente los requerimientos y el consumo de Se estarían asociados con la producción de leche y los días en lactancia al momento de la recolectar las muestras.

Estos resultados merecen una cuidadosa interpretación, ya que las prácticas de suplementación con Se han cambiado sustancialmente desde cuando los valores referenciales fueron establecidos. Muchos de estos valores fueron obtenidos usando suplementos inorgánicos (e.g. selenito de sodio), mientras que en años recientes se ha venido usando el Se orgánico con mayor frecuencia (Ceballos y col 2009). Suministrando el Se a la recomendación actual (i.e. $0,3 \mathrm{mg} / \mathrm{kg}$ base materia seca) y reemplazando la forma inorgánica por orgánica la concentración de Se en leche puede ser el doble (Ceballos y col 2009), mientras que el Se plasmático o sanguíneo se incrementa sólo entre un 10 y $20 \%$. Los valores bajos y marginales observados en este estudio pueden reflejar mejor el contenido de Se en la dieta que las prácticas de suplementación como tal, ya que estos valores no coinciden con lo reportado en la literatura. Los valores más altos podrían estar indicando diferentes prácticas de suplementación en esos predios, pero la evaluación del contenido de Se en la dieta y las prácticas de suplementación no fueron el objeto de este estudio, requiriéndose estudios complementarios.

La concentración de Se en la leche del estanque de frío no se asoció con los parámetros productivos estudiados. Pese a haberse indicado que la producción de leche puede ser un indicador sensible del balance de Se (Fraser y col 1987, Ellison 1992), otros estudios previos son coincidentes en señalar que no hay asociación entre el balance de Se e indicadores productivos, como la producción de leche (Kommisrud y col 2005, Enjalbert y col 2006, Ceballos-Márquez y col 2012). El efecto sobre proteína láctea no fue significativo después de controlar por la época del año $(\mathrm{P}=0,70)$, indicando que esta variable estaba confundiendo la asociación (Dohoo y col 2009). La observación de una asociación positiva entre la concentración de Se en leche y la producción de leche puede ser una relación no real, ya que los predios con mayor producción media pueden tener un manejo más complejo caracterizado por una mayor suplementación con Se, con el objeto de lograr algunos de los beneficios de administrar más de este mineral. Igualmente, no debe dejarse de lado el efecto de la estacionalidad de los partos, lo que puede afectar la cantidad de leche producida y en consecuencia el requerimiento de $\mathrm{Se}$.

El hecho de no observar una asociación entre la concentración de Se en leche con el RCS en el estanque, contradice un número de estudios que sugieren un rol del Se en la respuesta inmune de la glándula mamaria (Smith y col 1997, Sordillo y Aitken 2009). No obstante, estos resultados son coincidentes con otros estudios donde no se observó una asociación entre estas dos variables (Wichtel y col 2004, Ceballos y col 2010, Ceballos-Márquez y col 2012). Claramente, esto es una indicación de que tratándose de una variable de naturaleza multifactorial el manejo general del rebaño (e.g. otros nutrientes presentes en la dieta, procedimientos de ordeño adecuados, etc.) y el medio ambiente son factores determinantes de la ocurrencia de infecciones intramamarias, por lo que el estado nutricional de Se no sería el factor más importante en determinar el RCS en el estanque bajo las condiciones en los rebaños estudiados (Wichtel y col 2004).

La presencia de otros nutrientes en la dieta de animales en pastoreo (e.g. vitamina $\mathrm{E}$ y otros antioxidantes) podría suplir parcialmente el requerimiento de Se (Wichtel y col 1996). Muchas vacas mantenidas en pastoreo pueden tener un consumo adecuado de vitamina $E$ dado que el consumo de forrajes frescos es mayor comparado con vacas confinadas, lo que puede tener un efecto sobre el requerimiento de Se; pero la magnitud de este reemplazo no ha sido adecuadamente cuantificada (NRC 2001) y puede estar también afectada por el consumo de ácidos grasos en la ración (Wichtel y col 1996). Claramente, cuando el consumo de Se es más bajo de lo requerido, la vitamina E puede tener un efecto en la incidencia y severidad de las infecciones intramamarias (Weiss y col 1997).

En conclusión, los resultados obtenidos en este estudio indican la necesidad de una mayor suplementación con $\mathrm{Se}$ para lograr una leche con una concentración de Se cercana a lo observado en otros países, lo que traería consigo mejorar el aporte y la biodisponibilidad de Se en la dieta para los consumidores. Tener productos diseñados según la demanda del consumidor sería un nicho de mercado atractivo para las empresas procesadoras de lácteos. No obstante, el efecto del mayor consumo de Se sobre la productividad de la vaca y la sanidad mamaria no está del todo claro según los resultados obtenidos. Esta discrepancia con resultados previos puede deberse a la presencia de otros factores en la dieta que suplirían parcialmente el bajo contenido de Se de la pradera, así como también puede ser que las vacas mantenidas en sistemas pastoriles tengan un requerimiento diferente al sugerido para vacas en sistemas confinados, lo que requiere de la realización de estudios posteriores. 


\section{RESUMEN}

El objetivo de este estudio fue evaluar la concentración de selenio (Se) en muestras de leche procedentes de rebaños lecheros del sur de Chile y sus eventuales variaciones estacionales, y complementariamente examinar la eventual relación entre dicha concentración con algunos indicadores de producción. Para ello se seleccionaron 59 rebaños lecheros del sur de Chile, en los que se obtuvieron muestras de leche del estanque predial en cuatro oportunidades, primavera 2007 y verano, otoño e invierno del 2008 para determinar la concentración de Se en leche entera. Además, se colectó información del manejo, de la nutrición mineral en el predio y de indicadores de producción de leche, como producción media, contenido de grasa, proteína y recuento de células somáticas. Los datos fueron evaluados mediante modelos mixtos de regresión lineal. La concentración media de Se en leche del estanque fue 14,7 $\mu \mathrm{g} / \mathrm{L}$ (DE: $6,5 \mu \mathrm{g} / \mathrm{L}$ ), señalando una elevada varianza entre rebaños $(44 \%)$, no observándose ninguna asociación con los parámetros productivos evaluados. Pese a lo anterior, un $82 \%$ del total de muestras colectadas $(\mathrm{n}=231)$ presentaron valores $<20 \mu \mathrm{g} / \mathrm{L}$. La concentración de Se en la leche de estanques de enfriamiento en rebaños lecheros seleccionados en el sur de Chile indica que las vacas presentan un inadecuado balance de $\mathrm{Se}$, que no necesariamente se ve reflejado en una baja producción o calidad sanitaria de la leche.

\section{REFERENCIAS}

Ali AKA, GE Shook. 1980. An optimum transformation for somatic cell concentration in milk. J Dairy Sci 63, 487-490.

Bauman DE, I Mather, R Wall, AL Lock. 2006. Major advances associated with the biosynthesis of milk. J Dairy Sci 89, 1235-1243.

Carver JD. 2003. Advances in nutritional modifications of infant formulas. Am J Clin Nutr 77 (Suppl.), 1550S-1554S.

Ceballos A, F Wittwer, PA Contreras, H Böhmwald. 1998. Actividad sanguínea de glutatión peroxidasa en rebaños lecheros a pastoreo: Variación según edad y época del año. Arch Med Vet 30, 13-22.

Ceballos A, FG Wittwer, PA Contreras, E Quiroz, H Böhmwald. 1999. Actividad de glutatión peroxidasa en bovinos lecheros a pastoreo correlacionada con la concentración sanguínea y plasmática de selenio. Pesq Agrop Bras 34, 2331-2338.

Ceballos A, J Sánchez, H Stryhn, JB Montgomery, HW Barkema, JJ Wichtel. 2009. Meta-analysis of the effect of oral selenium supplementation on milk selenium concentration in cattle. J Dairy Sci 92, 324-342.

Ceballos A, J Kruze, HW Barkema, IR Dohoo, J Sanchez, D Uribe, JJ Wichtel, F Wittwer. 2010. Barium selenate supplementation and its effect on intramammary infection in pasture-based dairy cows. J Dairy Sci 93, 1468-1477.

Ceballos-Márquez A, HW Barkema, H Stryhn, IR Dohoo, GP Keefe, JJ Wichtel. 2012. Bulk tank milk selenium and its association with milk production parameters in Canadian dairy herds. Can Vet J (In press).

Conrad HR, AL Moxon. 1979. Transfer of dietary selenium to milk. $J$ Dairy Sci 62, 404-411.

Dohoo I, W Martin, H Stryhn. 2009. Veterinary Epidemiologic Research. $2^{\text {nd }}$ ed. VER Inc, Charlottetown, Canada.

Ellison RS. 1992. A review of copper and selenium reference ranges in cattle and sheep. Vet Cont Educ 145, 3-27.

Enjalbert F, P Lebreton, O Salat. 2006. Effects of copper, zinc and selenium status on performance and health in commercial dairy and beef herds: Retrospective study. J Anim Physiol Anim Nutr 90, 459-466.
Fraser AJ, TJ Ryan, R Sproule, RG Clark, D Anderson, EO Pederson. 1987. The effect of selenium supplementation on milk production in dairy cattle. Proc N Z Soc Anim Prod 47, 61-64.

Grace ND, K Ankebauer-Perkins, AM Alexander, RM Marchant. 2001. Relationship between blood selenium concentration or glutathione peroxidase activity, and milk selenium concentrations in New Zealand dairy cows. $N Z$ Vet $J 49,24-28$.

Knowles SO, ND Grace, K Wurms, J Lee. 1999. Significance of amount and form of dietary selenium on blood, milk, and casein selenium concentrations in grazing cows. J Dairy Sci 82, 429-437.

Knowles SO, ND Grace, TW Knight, WC McNabb, J Lee. 2004. Adding nutritional value to meat and milk from pasture-fed livestock. $N Z$ Vet $J$ 52, 342-351.

Kommisrud E, O Osteras, T Vatn. 2005. Blood selenium associated with health and fertility in Norwegian dairy herds. Acta Vet Scand $46,229-240$

Laporte JA. 1998. Actividad sanguínea de glutatión peroxidasa como indicador del balance metabólico nutricional de selenio en bovinos del área lechera de Los Ángeles, Centro-Sur de Chile. Memoria de título, Escuela de Medicina Veterinaria, Universidad Austral de Chile, Valdivia, Chile.

Miller, GY, PC Bartlett, RJ Erskine, KL Smith. 1995. Factors affecting serum selenium and vitamin E concentrations in dairy cows. $J$ Am Vet Med Assoc 206, 1369-1373.

Muñiz-Naveiro O, R Domínguez-González, A Bermejo-Barrera, JA Cocho de Juan, JM Fraga, A Goris, A López, I Martínez, J Valledor, L Fernández-Couto, P Bermejo-Barrera. 2005. Selenium content and distribution in cow's milk supplemented with two dietary selenium sources. J Agric Food Chem 53, 9817-9822.

National Research Council, NRC. 2001. Nutrient requirements of dairy cattle. $7^{\text {th }}$ ed. Natl. Acad. Sci., Washington DC, USA.

Norrman E. 1984. The selenium content of milk. Nord Vet-Med 36, 296-304.

Pfeuffer M, J Schrezenmeir. 2000. Bioactive substances in milk with properties decreasing risk of cardiovascular diseases. $\mathrm{Br} J$ Nutr 84, S155-159.

Smith K, J Hogan, W Weiss. 1997. Dietary vitamin E and selenium affect mastitis and milk quality. J Anim Sci 75, 1659-1665.

Sordillo LM, SL Aitken. 2009. Impact of oxidative stress on the health and immune function of dairy cattle. Vet Immunol Immunopathol 128, 104-109.

Weiss WP, JS Hogan, DA Todhunter, KL Smith. 1997. Effect of vitamin $\mathrm{E}$ supplementation in diets with a low concentration of selenium on mammary gland health of dairy cows. J Dairy Sci 80, 1728-1737.

Wichtel JJ, DA Freeman, AL Craigie, H Varela-Alvarez, NB Williamson. 1996. Alpha-tocopherol, selenium and polyunsaturated fatty acid concentrations in the serum and feed of spring-calving dairy heifers. $N Z$ Vet J 44, 15-21.

Wichtel JJ. 1998. A review of selenium deficiency in grazing ruminants. Part 1: New roles for selenium in ruminant metabolism. $N Z$ Vet $J$ 46, 47-52.

Wichtel JJ, GP Keefe, JA Van Leeuwen, E Spangler, MA McNiven, T Ogilvie. 2004. The selenium status of dairy herds in Prince Edward Island. Can Vet J 45, 124-132.

Wittwer F, P Araneda, A Ceballos, PA Contreras, M Andaur, H Böhmwald. 2002. Actividad de glutatión peroxidasa (GPx1) en sangre de bovinos a pastoreo de la IX Región, Chile y su relación con la concentración de selenio en el forraje. Arch Med Vet 34, 49-57. 